\title{
On the relation between the holomorphic prepotential and the quantum moduli in SUSY gauge theories *
}

\author{
J. Sonnenschein ${ }^{a}, S$. Theisen ${ }^{b}$, S. Yankielowicz ${ }^{a}$ \\ a School of Physics and Astronomy, Beverly and Raymond Sackler Faculty of Exact Sciences, \\ Tel-Aviv University, Ramat-Aviv, Tel-Aviv 69978, Israel \\ b Sektion Physik, Universität München, Theresienstraße 37, 80333 München, FRG
}

Received 30 October 1995

Editor: L. Alvarez-Gaumé

\begin{abstract}
We give a simple proof of the relation $\Lambda \partial_{\Lambda} \mathcal{F}=\frac{i}{2 \pi} b_{1}\left\langle\operatorname{Tr} \phi^{2}\right\rangle$, which is valid for $N=2$ supersymmetric QCD with massless quarks. We consider $S U\left(N_{c}\right)$ gauge theories as well as $S O\left(N_{c}\right)$ and $S P\left(N_{c}\right)$. An analogous relation which corresponds to massive hypermultiplets is written down. We also discuss the generalizations to $N=1$ models in the Coulomb phase.
\end{abstract}

A lot of activity has followed the beautiful work of Seiberg and Witten [1] on the exact non-perturbative low energy effective action (in the Coulomb phase) of the pure and QCD-like $S U(2) N=2$ supersymmetric gauge theories. In [2] it was generalized to $S U\left(N_{C}\right)$ $N=2$ theories and in $[3,4]$ to $S U\left(N_{C}\right) N=2$ theories with matter in the fundamental representation. Recently this work has been extended to $S O\left(N_{C}\right)$ and $S p\left(N_{C}\right)$ gauge groups [5-7].

In the present letter we prove and discuss relations between the prepotential $\mathcal{F}$ and the quantum moduli of the $N=2$ theory. The most interesting relation rcads

$\Lambda \frac{\partial}{\partial \Lambda} \mathcal{F}=\frac{i}{2 \pi} b_{1}\left\langle\operatorname{Tr} \phi^{2}\right\rangle$

where $\phi$ is the adjoint complex scalar in the $N=2$ gauge multiplet, and $b_{1}$ is the one-loop coefficient of the beta-function. This relation holds for all $N=2$

\footnotetext{
ऋ Work supported in part by GIF - the German-Israeli Foundation for Scientific Research.
}

theories, either pure or with massless matter quarks. For the case of pure $S U(2)$ this relation is essentially proven in [8] where the modular transformations of the prepotential $\mathcal{F}$ are considered. In [9] the generalization of the Seiberg-Witten approach to $N=2$ string theory is investigated. In particular, the exact nonperturbative result on pure $S U(2)$ and $S U(3) N=2$ Yang-Mills theory were recovered from the tree-level Type II string theory at the corresponding points in moduli space, in the limit of $\alpha^{\prime} \rightarrow 0$, where gravity is decoupled. In this work it was observed that starting from the local case $u \equiv \frac{1}{2}\left\langle\operatorname{Tr} \phi^{2}\right\rangle$ behaves as a period and the relation (1) holds with the dilaton playing the role of $\Lambda$. This relation turns out to be crucial in obtaining the rigid theory from the local one.

In the pure $N=2$ gauge theory, the low energy effective action up to terms with two derivatives is completely determined by one holomorphic function of $N=2$ chiral superfields $\mathcal{A}_{i}$, the prepotential $\mathcal{F}(\mathcal{A})$. For $N_{f}>0$, we also have to include (matter) hypermultiplets, whose contribution to the low energy 
effective action is not determined by a holomorphic structure. However, for the purpose of this note, we won't need their couplings. For the massless case, the perturbative piece of the prepotential is

$$
\begin{aligned}
& \left(\frac{(\boldsymbol{\alpha} \cdot \mathcal{A})^{2}}{\Lambda^{2}}\right) \mathcal{F}_{\text {pert. }}(\mathcal{A})=\frac{1}{2 \pi i} \frac{l(\text { adj. })-\sum_{i} l_{i}(\text { matter })}{l(\text { adj. })} \\
& \quad \times \sum_{\alpha>0}(\boldsymbol{\alpha} \cdot \mathcal{A})^{2} \ln \left(\frac{(\boldsymbol{\alpha} \cdot \mathcal{A})^{2}}{\Lambda^{2}}\right)
\end{aligned}
$$

The sum is over all positive roots and $l$ (adj.) is the index of the adjoint representation of the gauge group $G$ whereas $l_{i}$ (matter) is the index of the representation of the $i$ th matter hypermultiplet. From this expression the perturbative beta-function, which is purely oneloop, follows.

The prepotential may be considered as a holomorphic function of the chiral superfields $\mathcal{A}_{i}$ and the scale $\Lambda$. Defining $a_{i}=\left.\mathcal{A}_{i}\right|_{\theta=0}$ and $a_{D_{i}}=\frac{\partial \mathcal{F}(a)}{\partial a_{i}}$, one then finds that $\left(a_{i}, a_{D_{i}}\right)$ are the periods of an abelian differential of the second kind (having poles with zero residue) for the case of $N_{f} \geq 0$ massless hypermultiplets or of the third kind (having poles with non-zero residue) for $N_{f}>0$ massive hypermultiplets. These differentials are defined on an (auxiliary) hyperelliptic Riemann surface $\Sigma_{r}$ of genus $r=\operatorname{rank}(G)$ and the periods are with respect to a symplectic homology basis with one-cycles $\left(\alpha_{i}, \beta_{i}\right)$. The Riemann surfaces for pure $S U\left(N_{C}\right)$ [2], $S U\left(N_{C}\right)$ with hypermultiplets $[3,4], S O\left(N_{C}\right)$ without [5] and with [6,7] matter, and finally also for $S p\left(N_{C}\right)$ [7] have been found by now. In particular Ref. [7] gives curves with genus equal to the rank of $G$. The hypermultiplets were always chosen in the defining representation and their number such that the theory is either asymptotically free or has vanishing beta function. Recently curves for certain $N=1$ supersymmetric theories were considered in $[10,4,11]$ with matter in the adjoint and/or fundamental representations. We first treat $N=2$ theories with $G=S U\left(N_{C}\right)$. The remaining classical groups and some $N=1$ cases will be dealt with below.

The Riemann surface for $S U\left(N_{C}\right)$ is the genus $N_{C}-$ 1 hyperelliptic curve $\Sigma_{N_{C}-1}$

$$
y^{2}=W^{2}+F
$$

where
$W=\langle\operatorname{det}(x \mathbf{1}-\phi)\rangle \equiv x^{N_{C}}-\sum_{k=2}^{N_{C}} s_{k} x^{N_{C}-k}$

$F=F\left(x, m_{j}, \Lambda\right)$ is a polynomial of its arguments, independent of the $s_{i}$ and $F(x) \sim x^{N_{f}}$ for large $x$. If we parametrize $\langle\phi\rangle=\sum_{i} a_{i} H_{i}$ where $H_{i}$ are the generators in the Cartan subalgebra, we get in the semiclassical limit $s_{2}=\frac{1}{2} a_{i} a_{j} \operatorname{Tr}\left(H_{i} H_{j}\right)$. The exact (nonperturbative) expression is $s_{2}=u=\frac{1}{2}\left\langle\operatorname{Tr} \phi^{2}\right\rangle$ where $\phi$ is the Higgs field, i.e. the scalar component of the $N=1$ chiral superfield contained in the $N=2$ chiral superfield.

The meromorphic differential $\lambda$ is $[3,4]^{\prime}$ (the prime denotes differentiation w.r.t. $x$ )

$\lambda=\frac{1}{2 \pi i}\left(W F^{\prime}-2 F W^{\prime}\right) \frac{(x+b) d x}{F y}$

where the normalization is chosen such that $(i=$ $\left.1, \ldots, N_{C}-1\right) a_{i}=\int_{\alpha_{i}} \lambda, a_{D_{i}}=\int_{\beta_{i}} \lambda$ and $\partial_{s_{k}} a_{i}=$ $\int_{\alpha_{i}} \omega_{k}, \partial_{s_{k}} a_{D_{i}}=\int_{\beta_{i}} \omega_{k} \cdot \omega_{k}=\frac{\partial}{\lambda_{s_{k}}} \lambda=\frac{1}{\pi i} \frac{x^{N} C^{-k} d x}{y}, k=$ $2, \ldots, N_{C}$, are a basis of holomorphic differentials (abelian differentials of the first kind) on $\Sigma_{N_{C}-1}$. The constant $b=b(\Lambda, m)$ must be chosen such that for the massless case there are no polcs at zeroes of $F$ and the pole at infinity has zero residue. In the massive case $\lambda$ must have poles at the zeroes of $F$ with residues $m_{j}$. One finds that in the massless case $b=0 . \lambda$ also has a double pole at infinity with residue $-\sum m_{j}$ which vanishes in the massless case. It is, therefore, an abelian differential of the second and third kind in the massless and massive cases, respectively.

The effective (field dependent, dimensionless) gauge coupling is given by the matrix $\tau_{i j}=\frac{\partial^{2} \mathcal{F}}{\partial a_{i} j a_{j}}$. $\mathcal{F}$ is thus a homogeneous function of weight two of $a_{i}, m_{j}, \Lambda$ and satisfies the Euler equation ${ }^{2}$

$2 \mathcal{F}=\left(\Lambda \partial_{\Lambda}+\sum_{j} m_{j} \partial_{m j}+\sum_{i} a_{i} \partial_{a_{i}}\right) \mathcal{F}$

Taking derivatives w.r.t. to $s_{k}$ and using the definition of the $a_{D_{i}}$ one obtains

\footnotetext{
${ }^{1}$ Here and below relations between abelian differentials are always up to exact differentials.

${ }^{2}$ Here and below, $\Lambda$ is always meant to be $\Lambda_{N}$.
} 


$$
\begin{aligned}
& \frac{\partial}{\partial s_{k}}\left(\Lambda \partial_{\Lambda}+\sum_{j} m_{j} \partial_{m_{j}}\right) \mathcal{F} \\
& =\sum_{i}\left(a_{i} \frac{\partial}{\partial s_{k}} a_{D_{i}}-a_{D_{i}} \frac{\partial}{\partial s_{k}} a_{i}\right)
\end{aligned}
$$

Using now the above results we arrive at

$$
\begin{aligned}
& \frac{\partial}{\partial s_{k}}\left(\Lambda \partial_{\Lambda}+\sum_{j} m_{j} \partial_{m_{j}}\right) \mathcal{F} \\
& \quad=\sum_{i} \int_{\alpha_{i}} \lambda \int_{\beta_{i}} \omega_{k}-\int_{\beta_{i}} \lambda \int_{\alpha_{i}} \omega_{k}
\end{aligned}
$$

The right hand side of this equation can be evaluated with the help of a Riemann bilinear relation [12]. Since they make a distinction between $\lambda$ being abelian of second or third kind, we will treat the massless and massive cases separately. We first discuss the massless case, where the integrals on the right hand side of Eq. (8) can be done explicitly. The mass dependent terms on the left hand side of Eqs. (7) and (8) are now absent and $\lambda$ has a double pole at $w=1 / x=0$ with expansion

$\lambda=\left(\lambda_{-2} w^{-2}+\lambda_{0}+\lambda_{1} w+\ldots\right) d w$

with $\lambda_{-2}=\frac{1}{2 \pi i}\left(2 N_{C}-N_{f}\right)$; there are no further poles of $\lambda$. The Riemann bilinear relation now reads

$$
\begin{gathered}
\sum_{i} \int_{\alpha_{i}} \lambda \int_{\beta_{i}} \omega_{k}-\int_{\beta_{i}} \lambda \int_{\alpha_{i}} \omega_{k} \\
=2 \pi i \sum_{n \geq 2} \frac{\lambda_{-n} \omega_{n-2}^{(k)}}{n-1}
\end{gathered}
$$

where $\omega_{n}^{(k)}$ are the coefficients of $\omega_{k}$ in its expansion around infinity:

$$
\begin{aligned}
\omega_{k} & =\left(\omega_{0}^{(k)}+\omega_{1}^{(k)} w+\ldots\right) d w \\
& =\left(-\frac{1}{i \pi} w^{k-2}+O\left(w^{k-1}\right)\right)
\end{aligned}
$$

i.e. $\omega_{0}^{(k)}=-\frac{1}{\pi i} \delta_{k, 2}$. We then have

$$
\begin{gathered}
\partial_{s_{k}}\left(\Lambda \partial_{\Lambda} \mathcal{F}\right)=2 \pi i \lambda_{-2} \omega_{0}^{(k)} \\
=\frac{i}{\pi}\left(2 N_{C}-N_{f}\right) \delta_{k .2}
\end{gathered}
$$

Integration gives
$\Lambda \partial_{\Lambda} \mathcal{F}=\frac{i}{\pi}\left(2 N_{C}-N_{f}\right) s_{2}$

where comparison with the weak coupling expression shows that a possible contribution const. $\Lambda^{2}$ is absent from the right hand side. Let us briefly comment on this result. Taking derivatives with respect to $a_{i}$ and $a_{j}$ and using the definition $\partial_{a_{i}} \partial_{a_{j}} \mathcal{F}=\tau_{i j}=\frac{1}{2 \pi} \theta_{i j}+$ $4 \pi i\left(\frac{1}{g^{2}}\right)_{i j}$ one obtains

$$
\begin{aligned}
& \Lambda \frac{d}{d \Lambda} \tau_{i j}=\frac{i}{2 \pi}\left(2 N_{C}-N_{f}\right) \partial_{a_{i}} \partial_{a_{j}} \operatorname{Tr}\left\langle\phi^{2}\right\rangle \\
& \simeq \frac{i}{\pi}\left(2 N_{C}-N_{f}\right) \operatorname{Tr}\left(H_{i} H_{j}\right)
\end{aligned}
$$

where in the last step we have taken the semi-classical limit, i.e. have suppressed instanton corrections.

We note that the relation (14) is compatible with perturbation theory. It is well known [13] that $\mathcal{F}$ (or, equivalently, the Wilsonian field dependent gauge coupling) acquires a contribution only at one loop level. This means that $s_{2}$ is equal (up to nonperturbative contributions) to its classical value. This agrees with the general observation that correlators of lowest components of gauge invariant chiral superfields are "topological", i.e. they do not depend on positions [14]. Thus they get contributions only from disconnected diagrams. Moreover, they depend holomorphically on the parameters, notably on the gauge coupling. This in fact implies (since there is no dependence on $\theta$ in perturbation theory) that there are no perturbative quantum corrections to the classical result. Note, however, that the exact beta function is proportional to $\partial_{a_{i}} \partial_{a_{j}}\left\langle\operatorname{Tr} \phi^{2}\right\rangle$, which includes instanton corrections. The above discussion also applies to all the other invariants $s_{k}$, and the absence of logarithms, which would have appeared in perturbative contributions, is necessary for them to be globally defincd coordinates on the quantum moduli space.

Let us now turn to the remaining classical groups with $N_{f}$ hypermultiplets in the defining representation $\underline{N}_{C}$ [7]. Here the Riemann surfaces are given by curves of the form [7]

$x y^{2}=W^{2}+F$

where now for $x \rightarrow \infty, W \sim x^{r}$ and $F \sim x^{N_{f}+\nu}$ where $\nu=4,3,0$ for $S O(2 r), S O(2 r+1)$ and $S p(2 r)$, respectively. The meromorphic differential $\lambda$ is 
$\lambda=\frac{1}{2 \pi i} \frac{W F^{\prime}-2 W^{\prime} F}{y F} d x$

with the asymptotic behavior at infinity $\lambda \sim$ $\frac{1}{2 \pi i}\left(l(\right.$ adj. $\left.)-N_{f} l\left(\underline{N}_{C}\right)\right) \frac{d x}{\sqrt{x}}$ where $l(\operatorname{adj})=.2\left(N_{C}-\right.$ 2), $N_{C}+2$ and $l\left(\underline{N}_{C}\right)=2,1$ for $S O\left(N_{C}\right)$ and $S p\left(N_{C}\right)$, respectively. The combination of the indices of the representations appearing in the asymptotic expression of $\lambda$, is exactly the one-loop coefficient $b_{1}$ of the beta-function for an $N=2$ supersymmetric gauge theory with $N_{f}$ hypermultiplets in the defining representation. Introducing the local uniformization variable $x=1 / \xi^{2}$ one finds that (we are again only considering the massless case here)

$\lambda_{-2}=-\frac{1}{\pi i}\left(l(\operatorname{adj})-.N_{f} l\left(\underline{N}_{C}\right)\right)$

Likewise one finds the asymptotic behavior of $\omega_{k}=$ $\partial_{s_{k}} \lambda$ as $\omega_{k}=\left(\omega_{0}^{(k)}+\omega_{1}^{(k)} \xi+\ldots\right) d \xi$ with $\omega_{0}^{(k)}=$ $-\frac{1}{\pi i} \delta_{k, 1}$. Note that in the notation of Ref. [7] $s_{1}$ is the quadratic invariant: $s_{1}=\frac{1}{2} \operatorname{Tr}\left\langle\phi^{2}\right\rangle$. Inserting this into the Riemann relation ( 8 ) we get

$$
\frac{\partial}{\partial s_{k}}\left(\Lambda \partial_{\Lambda} \mathcal{F}\right)=\frac{2 i}{\pi}\left(l(\operatorname{adj} .)-N_{f} l\left(\underline{N}_{C}\right) \delta_{k, 1}\right)
$$

Let us now turn to the massive case. Here we have to use the Riemann bilinear relation for one abelian differential of the first kind $\left(\omega_{k}\right)$ and the other of the third kind $(\lambda)$ with first and second order poles. We will concentrate on the case of $S U\left(N_{C}\right)$. The other groups can be treated similarly. In fact, the meromorphic differential $\lambda$ now has simple poles at $x_{i}=m_{i}$ with residues $m_{i}$ and a double pole at infinity where it behaves as

$$
\lambda=\left(\lambda_{-2} w^{-2}+\lambda_{-1} w^{-1}+\lambda_{0}+\ldots\right) d w
$$

with

$$
\lambda_{-2}=\frac{1}{2 \pi i}\left(2 N_{C}-N_{f}\right), \quad \lambda_{-1}=-\frac{1}{2 \pi i} \sum_{i=1}^{N_{f}} m_{i}
$$

The relevant bilinear relation gets contributions from both of these coefficients as well as from the residues of the poles at $x_{i}=m_{i}$. The contribution from $\lambda_{-2}$ is the same as in the massless case. The contribution from the poles at $m_{i}$ and the pole at infinity is

$$
\begin{aligned}
& 2 \pi i \sum_{i} \operatorname{res}_{x_{i}} \lambda \int_{x_{0}}^{x_{i}} \omega_{k}+2 \pi i \operatorname{res}_{\infty} \lambda \int_{x_{0}}^{\infty} \omega_{k} \\
& =-\sum_{i=1}^{N_{f}} m_{i} \int_{m_{i}}^{\infty} \omega_{k}
\end{aligned}
$$

where $x_{0}$ is an arbitrarily chosen point on the Riemann surface $^{3}$. This leads to

$$
\begin{aligned}
& \frac{\partial}{\partial s_{k}}\left(\Lambda \partial_{\Lambda}+\sum_{i} m_{i} \partial_{m_{i}}\right) \mathcal{F}=\frac{i}{\pi}\left(2 N_{C}-N_{f}\right) \delta_{k, 2} \\
& \quad-\sum_{i} m_{i} \int_{m_{i}}^{\infty} \omega_{k}
\end{aligned}
$$

Recall that $\omega_{k}=\partial_{s_{k}} \lambda$ so that this relation can be integrated w.r.t. $s_{k}$ leading to a generalization of Eq. (13):

$$
\begin{aligned}
& \left(\Lambda \partial_{\Lambda}+\sum_{i} m_{i} \partial_{m_{i}}\right) \mathcal{F}=\frac{i}{2 \pi}\left(2 N_{C}-N_{f}\right)\left\langle\operatorname{Tr} \phi^{2}\right\rangle \\
& \quad-\sum_{i} m_{i} \int_{m_{i}}^{\infty} \lambda
\end{aligned}
$$

Note that now, in contrast to the massless case, the right hand side seems to depend on all the moduli $s_{k}$. We have not attempted to do the remaining integrals explicitly. But let us demonstrate that this expression has in fact the correct decoupling limit. We decouple one of the hypermultiplets by taking the limits, say, $m_{N_{f}} \equiv M \rightarrow \infty, \Lambda_{N_{f}} \rightarrow 0$ while keeping $\Lambda_{N_{f}-1}^{N_{C}-N_{f}+1}=M \Lambda_{N_{f}}^{N_{C}-N_{f}}$ fixed. To perform the integral $-M \int_{M}^{\infty} \omega_{k}$ we first change variables $x=M \tilde{x}$ and then perform the decoupling limit. In this limit $y(x) \rightarrow$ $M^{N_{C}} x^{N_{C}}$ and the integral becomes $\frac{i}{\pi} M^{2-k} \int_{1}^{\infty} \frac{d \tilde{x}}{\tilde{x}^{k}} \rightarrow$ $\frac{i}{\pi} \delta_{k, 2}$. The integrals for $i=1, \ldots, N_{f}-1$ only change in such a way that $\omega_{k}$ turns into the holomorphic differential appropriate for the curve with $N_{f}-1$ flavors. We thus find that on the right hand side of Eq. (21) we get the change $\left(2 N_{C}-N_{f}\right) \rightarrow\left(2 N_{C}-\left(N_{f}-1\right)\right)$. The left hand side changes as $\Lambda_{N_{f}} \partial_{\Lambda_{N_{f}}}+\sum_{i=1}^{N_{f}} m_{i} \partial_{m_{i}} \rightarrow$ $\Lambda_{N_{f}-1} \partial_{\Lambda_{N_{f}-1}}+\sum_{i=1}^{N_{f}-1} m_{i} \partial_{m_{i}}$.

\footnotetext{
${ }^{3}$ The independence of the choice follows from the fact that the residues of meromorphic differentials on Riemann surfaces sum up to zero. For more details on this relation, see Refs. [12].
} 
Let us now briefly mention that in all cases where $\Lambda \partial_{\Lambda} \mathcal{F}$ is proportional to $u, u$ is in fact invariant un$\operatorname{der} S p(2 r ; Z)$ transformations $\left(\begin{array}{c}a_{D} \\ a\end{array}\right) \rightarrow\left(\begin{array}{c}\tilde{a}_{D} \\ \tilde{a}\end{array}\right)=$ $\left(\begin{array}{ll}A & B \\ C & D\end{array}\right)\left(\begin{array}{c}a_{D} \\ a\end{array}\right)$. This is essentially proven in [8] for $S U(2)$. A simplified version of his proof can be easily generalized to arbitrary groups. From $\tilde{\mathcal{F}}(\tilde{a})=$ $\tilde{\mathcal{F}}(\tilde{a}(a))$ it follows that $\partial_{a^{j}} \tilde{\mathcal{F}}(\tilde{a}(a))=\left(\frac{\partial \tilde{a}^{i}}{\partial a^{j}}\right) \tilde{a}_{D_{i}}$. This relation can be integrated to yield

$$
\begin{aligned}
& \tilde{\mathcal{F}}(\tilde{a})=\mathcal{F}(a)+\frac{1}{2} a^{T} B^{T} D a+\frac{1}{2} a_{D}^{T} C^{T} A a_{D} \\
& \quad+a^{T} B^{T} C a_{D}
\end{aligned}
$$

This implies that $\mathcal{F}-\frac{1}{2} a^{T} a_{D}=\frac{1}{2} \Lambda \partial_{\Lambda} \mathcal{F}$ is invariant.

Finally, one may consider $N=2$ models in their Coulomb phase also for matter superfields in representations other than the adjoint or fundamental representations. For those cases it is plausible that the $b_{1}$ factor in Eq. (22) will be replaced by $2 N_{c}-\sum_{i} l_{i}$ (matter).

The gauge kinetic terms of the low energy effective action of supersymmetric gauge theories in their Coulomb phase can be determined from hyperelliptic curves not only for $N=2$ supersymmetric models but also for $N=1$ ones [10]. As in the $N=2$ case, the ground state of these $N=1$ models is described by an hyperelliptic quantum moduli space characterized by its singularities and monodromies. The determination of the curve follows from the classical singularities, instantons corrections and the global symmetries of the theory. For instance the curves which correspond to $S U\left(N_{C}\right) N=1$ models with one adjoint representation and $N_{f}$ fundamentals (denoted by $\left(N_{\text {ad }}=1, N_{f}\right)$ ) takes the form of Eq. (3) [4]. The corresponding polynomial $F$ is given now by $F=$ $F\left(x, \Lambda, m_{i j}, Y_{i j}\right)$ where $m_{i j}$ and $Y_{i j}$ are the quark mass matrix and the matrix of Yukawa couplings. When $Y$ is a unit matrix and $m$ is diagonal the model admits an additional supesymmetry. The curves in that case turn into those of $N=2$ models with $N_{f}$ hypermultiplets.

Starting with a curve that corresponds to a given $N=1$ model in its Coulomb phase one can follow the same steps taken above and prove an analogous relation to the one given in Eq. (22). We now discuss the relation for certain $N=1$ classes of models. Using the curves of [4], it turns out that for the class of models $\left(1, N_{f}\right)$ the relation is the same as that given in Eq. (22) apart from a replacement of $m_{i}$ by the eigenvalues of the matrix $Y^{-1} m$.

In case of $(2,0) N=1$ models the condition for a Coulomb phase is that the determinant of the adjoint mass matrix vanishes [10]. The curve for $S U\left(N_{C}=\right.$ 2) [10] is identical to that of the $N=2$ case with $N_{f}=$ 0 when one replaces $\Lambda_{N=2}^{2}$ with $\frac{1}{2} \Lambda_{N=1} m_{\text {ad }}$. where $m_{\mathrm{ad}}$ is the mass of the massive adjoint superfield. A similar situation occurs in the $(2,1)$ model [11]. We therefore anticipate that the $\left(2, N_{f}\right)$ curves will coincide with those of the $\left(1, N_{f}\right)$ models by a substitution of $\Lambda_{N_{\mathrm{ad}=1}}^{2 N_{\mathrm{c}}-N_{f}} \sim \tilde{\Lambda}_{N_{\mathrm{ad}}=2}^{N_{\mathrm{c}}-N_{f}} m_{\mathrm{ad}}^{N_{c}}$.

Naively, it seems that the 1.h.s. of the relation, for instance for $N_{f}=0$, takes the form of $\tilde{\Lambda} \partial_{\tilde{\Lambda}} \mathcal{F}+m_{\mathrm{ad}} \partial_{m_{\mathrm{ad}}} \mathcal{F}$, and on the r.h.s. the term proportional to $s_{2}$ involves the $b_{1}$ pertaining to the one adjoint case. This is quite surprising since a priori we expect such a $b_{1}$ to appear only when the massive adjoint decouples. The full determination of the relation and the decoupling for this class of models as well as those which involve other representations is under current investigation.

The relation discussed in this paper appears as a simple partial differential equation for the prepotential $\mathcal{F}$. In order to determine $\mathcal{F}$ completely one needs more equations. Already in the pure $S U(2)$ case one needs one more independent relation. It would be great if one could obtain enough relations which would, in turn, determine $\mathcal{F}$ in a simple way.

Finally we note that while the local counterpart of this relation seems to be quite important [9], the full physical meaning of the relation still alludes us. For fixed $\Lambda$, in the massless case, we can rewrite it as

$$
\left(\sum_{i} a_{i} \partial_{a_{i}}-2\right) \mathcal{F}=\frac{1}{2 \pi i} b_{1}\left\langle\operatorname{Tr} \phi^{2}\right\rangle
$$

This equation looks completely quantum mechanical. Moreover, as discussed in this letter, its non-trivial content is associated with the non-perturbative contributions on both sides. The left hand side of (24) looks as if it is related to the "anomalous dimension" of $\mathcal{F}$, i.e. to the deviation of $\mathcal{F}$ from its classical dimension 2. This is due to quantum effects associated with the appearance of $\Lambda$. The right hand side involves the beta function. It is tempting to think that one could understand this relation in terms of RG ideas. So far we have not been successful in doing it.

One of us (S.T.) would like to thank F. Ferrari and $\mathrm{W}$. Lerche for valuable discussions and Tel Aviv 
University for hospitality. S.Y. would like to thank G. Veneziano for interesting discussions.

\section{References}

[1] N. Seiberg and E. Witten, Nucl. Phys. B 426 (1994) 19; B 431 ( 1994) 484.

[2] A. Klemm, W. Lerche, S. Theisen and S. Yankielowicz, Phys. Lett. B 344 (1995) 169; hep-th/941 2158;

C.P. Argyres and A. Farragi, Phys. Rev. Lett. 74 (1995) 3931;

A. Klemm, W. Lerche and S. Theisen, hep-th/9505150.

[3] P.C. Argyres, R. Plesser and A. Shapere, hep-th/9505100; J. Minahan and D. Nemeshansky, hep-th/9507032.

[4] A. Hanany and Y. Oz, hep-th/9505075.

[5] U. Danielson and B. Sundborg, hep-th/9504102; A. Brandhuber and K. Landsteiner, Phys. Lett. B 358 (1995) 73.
[6] A. Hanany, hep-th/9509176.

[7] P.C. Argyres and A. Shapere, hep-th/9509175.

[8] M. Matone, hep-th/9506102 and hep-th/9506181.

[9] S. Kachru, A. Klemm, W. Lerche, P. Mayr and C. Vafa, hep-th/9508155.

[10] K. Intriligator and N. Seiberg, Nucl. Phys. B 431 (1994) 551; B 444 (1995) 125.

[11] S. Elitzur, A. Forge, A. Giveon and E. Rabinovici, Phys. Lett. B 353 (1995) 79; hep-th/9509130.

[12] See e.g. P. Griffiths and J. Harris, Principles of Algebraic Geometry, John Wiley \& Sons, 1978, Chap. 2.2;

G. Springer, Introduction to Riemann Surfaces, AddisonWesley, 1957, Chap. 10.3.

[13] M. Shifman and A. Vainshtein, Nucl. Phys. B 359 (1991) 571.

[14] V. Novikov, M. Shifman, A. Vainshtein and V. Zakharov, Nucl. Phys. B 229 (1983) 381, 407;

G.C. Rossi and G. Veneziano, Phys. Lett. B 138 (1984) 195. 